\title{
Inhaltsübersicht
}

Vorwort $\quad \mathrm{V}$

Abkürzungsverzeichnis $\quad \mathrm{XI}$

$\begin{array}{lll}\text { Literaturverzeichnis } & \text { XIII }\end{array}$

Erstes Kapitel 1

Die Problematik der condicio-sine-qua-non-Formel 1

\section{Zweites Kapitel}

Notwendigkeit und Grenzen einer besonderen juristischen 12

$\begin{array}{lr}\text { Schadenszurechnung } & 12\end{array}$

$\begin{array}{ll}\text { Drittes Kapitel } & 16\end{array}$

Das Vermeidbarkeitsprinzip: Grundlagen $\quad 16$

I. Allgemeine Voraussetzungen des Vermeidbarkeits- 16 prinzips

II. Die condicio-sine-qua-non-Formel als Vermeid- 19 barkeitsformel 20

III. Vermeidbarkeitsprinzip und 20 Rechtswidrigkeitszusammenhang

Viertes Kapitel $\quad 22$

Stufen der Vermeidbarkeit: Die Vernichtung objektiver 22

Rettungsmöglichkeiten

I. Mangelnde Reichweite der condicio-sine-qua-non- 22

Formel

II. Die Vernichtung objektiver Rettungsmöglichkeites 23

III. Objektive und andere Rettungsmöglichkeiten 28 
Fünftes Kapitel 31

Das hypothetische Geschehen und die alternative Konkurrenz 31

I. Das erlaubte Verhalten des Schädigers 31

II. Die hypothetische Eigenschädigung des Opfers 33

III. Das hypothetische Verhalten eines Dritten 38

IV. Hypothetische Naturereignisse 41

V. Versichertes oder sonst gedecktes hypothetisches 41 Geschehen

VI. Hypothetisches unerlaubtes Verhalten des Schädigers 41

VII. Unmittelbarer Ersatz des Tatgeschehens durch das 41 hypothetische Geschehen; Bew eislast

VIII. Die gesetzliche Berücksichtigung des hypothetischen $\quad 47$ Geschehens und die Fälle des pflichtwidrig verschobenen Risikos

IX. Vermeidbarkeit und Gefährdungshaftung 49

X. Alternative Konkurrenz $\quad 50$

Sechstes Kapitel 53

Insbesondere: Das hypothetische Geschehen nach Eintritt eines 53

Schadenserfolgs

I. Zeitpunkt der Schadensberechnung 53

II. Differenzhypothese und Vermeidbarkeitsprinzip 53

III. Vermeidbarkeit und Vermeidepflicht 54

IV. Unmittelbarer Ersatz des Tatgeschehens durch das 56 hypothetische Geschehen

V. Gesetzlicher Ausschluß späteren hypothetischen

Geschehens; Enteignungsfälle; Lizenzgebühr;

Sachversicherung

VI. Leitsätze

Siebentes Kapitel $\quad 66$

Rechtsprechung $\quad 66$

I. BGH DAR 1960, 115 (Kausalität der Geschwindig- 66 keitsüberschreitung)

II. BGHZ 29, 393 (Schiffsmaklerfall) 67

III. BGH VersR 1957, 373 (Testamentsfall) 69

IV. BGH LM $\$ 287$ ZPO Nr. 10 (Selbstmordfall) 70

V. BGH LM $\ 823$ BGB (Ga) Nr. 18 (Unterlassene 71 Warnung) 
VI. RG JW 1937, 2466 Nr. 25 (Verhindertes Gebor bei einer Versteigerung)

VII. RGZ 9, 416 (Unterlassene Mitteilung eines Verstei- $\quad 74$ gerungstermins)

VIII. BGHZ 2, 138 (Schiffskollision auf einem Fluß) 76

IX. BGH LM $\ 823$ BGB (Aa) Nr. 21 (Allergieanfall 77 und späterer Tod durch Blut- und Knochenmarkerkrankungen)

X. BGHZ 7, 287 (Amtspflichtverletzung eines Voll- 78 streckungsrichters und eines Gerichtsvollziehers)

XI. RG HRR 1935 Nr. 1008 (Verletztes Stillhalte- 80 abkommen)

XII. BGH LM $\$ 839$ BGB (D) Nr. 8 (Hypothetische 81 Gerichtsentscheidung)

XIII. BGH LM $\$ 839$ BGB (C) Nr. 47 (Hypothetische 83 Ermessensentscheidung einer Behörde)

XIV. BGH LM $₫ 839$ BGB (D) Nr. 1 (Ermessensentschei- $\quad 85$ dung einer Behörde)

XV. RGZ 147, 129 (Dreschsatzfall) 86

XVI. RGZ 163, 129 (Operation ohne Einwilligung des 89 Patienten)

XVII. BGH LM $\ 826$ BGB (Gb) Nr. 3 (Journalistenfall) 91

XVIII. BGH LM $₫ 249$ BGB (Ba) Nr. 12 (Vergiftete 92 Schweine)

XIX. BGH NJW 1967, 551 (Schleusenunfall) 93

XX. RGZ 144, 348 (Pflichtverletzung eines Aufsichtsrats) 98

XXI. RG JW 1916, 1116 Nr. 5 (Aufsichtspflicht eines 99 Vormundschafts. ichters)

XXII. OGHZ 1, 308 (Löschteichfall) 100

XXIII. BGHZ 20, 275 (Brandgassenfall) 103

XXIV. RGZ 102, 390 (Unzulässige Beschlagnahme von 105 Patenten im Kriege)

XXV. RGZ 156, 187 (Zur Abwehr eines Dammbruchs 106 geöffnete Schleuse)

XXVI. BGHZ 10, 6 (Direktorfall) 107

XXVII. OLG Stuttgart MDR 1956, 164 Nr. 147

(Verdienstausfall bei Schwangerschaft)

XXVIII. RGZ 1, 66 (Verdienstausfall bei Strafverbüßung) 110

XXIX. OLG Koblenz NJW 1962, 399 (Hypothetische 111

Freiheitsentziehung)

XXX. RGZ 68, 352 (Spätere Trunksucht)

112

XXXI. RGZ 141, 365 (Unterschlagene Mietgelder) 113

XXXII. RGZ 142, 8 (Wertverlust eines Bauernhofs) 115

XXXIII. RG DJ 1940, 1014 (Wertminderung von Aktien) 117

$\begin{array}{ll}\text { XXXIV. BGH JR 1952, } 70 \text { (Beschlagnahme eines Personen- } & 119\end{array}$ kraftwagens) 
XXXV. BGHZ 29, 207 (Berliner Nord-Südachse) 121

XXXVI. BGH LM $\$ 823$ BGB (C) Nr. 3 (Baufälliges Haus) 123

XXXVII. RGZ 95, 87 (Einziehung zum Kriegsdienst) 124

XXXVIII. OLG Düsseldorf MDR 1963, 47 (Schäferhund mit 125 späterer Staupe)

XXXIX. LG Hechingen NJW 1965, 1916 (Vergifteter Dackel) 128

XL. BGH LM $\ 249$ BGB $(\mathrm{Ba}) \mathrm{Nr} .15$ (Gesprengte 129 Hausruine)

XLI. RGZ 169, 117 (Arthritisfall) 132

XLII. BGH MDR 1964, 220 (Ưbermäßige Grundstücks- 134 immissionen)

XLIII. OLG Stuttgart NJW 1949, 585 Nr. 10 (Traktorfall) 136

XLIV. OLG Celle NJW 1949, 585 Nr. 11 (Beschlagnahmte 138 Spirituosen)

XLV. BGHZ 8, 288 (Entwendete Reichsmarknoten) 139

XLVI. BGH WM 1960, 21 (Tschechische Feldbahn- 140 lokomotiven)

XLVII. BAG AP $₫ 1$ TVG (Friedenspflicht) Nr. 2 (schleswig-holsteinischer Metallarbeiterstreik)

$\begin{array}{ll}\text { Achtes Kapitel } & 151\end{array}$

$\begin{array}{ll}\text { Schrifttum } & 151\end{array}$

I. Chance und Schadensbegriff 151

II. Uberholende Kausalität und Schadensbegriff 153

III. Überholende Kausalität und Forderungsrisiko 160

IV. Uberholende Kausalität und hypothetisches 164

haftungsbegründendes Geschehen

V. Uberholende Kausalität und Vorteilsausgleichung 166

VI. Uberholende Kausalität und Interessenabwägung 168

VII. Uberholende Kausalität und Verschuldensgrade $\quad 169$

VIII. Uberholende Kausalität und Kausalität 171

IX. Überholende Kausalität und Anlagefälle 175

$\begin{array}{ll}\text { Neuntes Kapitel } & 179\end{array}$

$\begin{array}{ll}\text { Zusammenfassung } & 179\end{array}$ 